\title{
Spatial scaling of Arctic sea ice deformation
}

\author{
H. L. Stern ${ }^{1}$ and R. W. Lindsay ${ }^{1}$ \\ Received 12 March 2009; revised 17 July 2009; accepted 4 August 2009; published 21 October 2009.
}

[1] Arctic sea ice deformation arises from spatial gradients in the ice velocity field. This deformation occurs across a wide range of spatial scales, from meters to thousands of kilometers. We analyze 7 years of sea ice deformation data from the RADARSAT Geophysical Processor System (RGPS) covering the western Arctic Ocean. We find that the mean deformation rate is related to the spatial scale over which it is measured according to a power law with exponent $\sim-0.2$, over a scale range from 10 to $1000 \mathrm{~km}$ (e.g., deformation rate doubles for a 30 -fold reduction in scale). Both the exponent and the deformation rate have distinct annual cycles. The exponent becomes more negative in summer as the ice pack weakens and internal stresses are not as readily transmitted over long distances. The deformation rate reaches a minimum in late winter when the ice pack is strongest. The deformation also exhibits considerable localization, in which the largest deformation rates are confined to smaller and smaller areas as the scale of measurement decreases. This supports a model for sea ice based on granular or fracture mechanics. The scaling exponent in the power law relationship tends to be larger in magnitude where the concentration of multiyear ice is low, consistent with a thinner and weaker ice pack. With decreasing multiyear ice in the Arctic and a thinning ice pack, an increase in the deformation rate has already been documented (from buoy data). However, the net effect of several deformation/thickness feedbacks is still uncertain.

Citation: Stern, H. L., and R. W. Lindsay (2009), Spatial scaling of Arctic sea ice deformation, J. Geophys. Res., 114, C10017, doi:10.1029/2009JC005380.

\section{Introduction}

[2] Sea ice covers the central Arctic Ocean in summer and winter, moving under the influence of wind, ocean currents, and internal stress gradients. Spatial differences in the sea ice velocity constitute deformation, which is typically concentrated along narrow fracture zones or leads. Sea ice deformation is an important process in the Arctic climate system: divergence creates open water, new ice growth in winter, heat and moisture fluxes to the atmosphere, and brine rejection to the ocean; convergence creates pressure ridges and keels, altering the air and water drag coefficients; and resistance to shear deformation restricts the mobility of the ice in narrow passages. Thus sea ice deformation helps to drive changes in the sea ice thickness distribution, the spatial extent of sea ice, and near-surface atmospheric and oceanic fluxes. Our interest is in characterizing the sea ice deformation rate across a wide range of spatial scales.

[3] Power-law scaling is a relationship of the form $y=c x^{b}$ that relates the property $y$ to the spatial scale $x$ over which it is measured, where $c$ and $b$ are constants. Rescaling $x$ and $y$ maintains the same power-law relationship with exponent $b$ (only the constant of proportionality $c$ changes). Sea ice has been observed to exhibit such power law behavior over many orders of magnitude, with respect to the floe size distribution

\footnotetext{
${ }^{1}$ Polar Science Center, Applied Physics Laboratory, University of Washington, Seattle, Washington, USA.

Copyright 2009 by the American Geophysical Union. 0148-0227/09/2009JC005380\$09.00
}

[Rothrock and Thorndike, 1984; Lindsay and Rothrock, 1995; Korsnes et al., 2004], the mean deformation rate [Marsan et al., 2004], and fracturing processes; see Weiss [2001, 2003] for extensive reviews. Sea ice is not unique in this respect; many phenomena in nature show self-similar or fractal scaling properties described by power laws [e.g., Mandelbrot, 1982; Turcotte, 1992]. The distinguishing feature of a power law is the invariance of the exponent with respect to changes in scale. There is no characteristic or intrinsic length scale; all scales are linked. Once established, the power law provides a way to compare measurements made at different scales.

[4] One measurement of sea ice deformation requires at least three simultaneous observations of sea ice velocity at different locations. The separation of these locations defines a spatial scale. With many observations of sea ice velocity across a broad area, one may construct many measurements of sea ice deformation, each with an associated spatial scale. For a given scale range, such as 100 to $200 \mathrm{~km}$, and many independent measurements of the deformation, one thus obtains a distribution of deformation measurements. The mean deformation $(y)$ within each scale range $(x-\Delta x, x+$ $\Delta x$ ) has previously been found to obey power-law scaling: $y=$ $c x^{b}$ where $b=-0.20 \pm 0.01$ [Marsan et al., 2004]. This was based on a single "snapshot" of sea ice velocities calculated from satellite data over a single 3-day period in November 1997.

[5] Marsan et al. [2004] also discussed the localization of deformation, finding that the largest $15 \%$ of the deformation occurs within a smaller and smaller area as the scale of 
measurement decreases. A similar result was obtained by Hopkins and Thorndike [2006], who constructed a granular model of sea ice that explicitly models the fracturing process between floes. They obtained a power-law floe size distribution, and they found that the damage zones between floes became more localized as the resolution of the simulated sea ice cover became finer. Their work offers a plausible mechanical theory for how the power-law floe size distribution and the localization of deformation might occur. Localization is discussed further in section 6 .

[6] Rampal et al. [2008] took a different approach to the scaling of sea ice deformation, using the change in separation of pairs of buoys deployed on the ice as a proxy for deformation. They found a spatial scaling law in which the exponent depends on the time interval over which the velocities are calculated, and which agrees with the exponent $b=-0.2$ for time scales of three days. Moreover, the deformation also obeyed power-law scaling in time, and exhibited localization (termed "intermittency" in the time domain) as the time scale decreased.

[7] Kwok [2006] examined Arctic sea ice deformation during 1997-2000 using the same source data that we use, from the RADARSAT Geophysical Processor System (RGPS). He partitioned the ice cover into a seasonal ice zone (SIZ, $\leq 80 \%$ multiyear ice) and a perennial ice zone (PIZ, $>80 \%$ multiyear ice). He found that the deformation rate is larger in the SIZ than in the PIZ, with both zones exhibiting an annual cycle with a minimum in midwinter and a maximum in late fall. Our analysis leads to similar results, which are further discussed in section 7.

[8] Sea ice models generally employ a momentum equation that treats the ice as a continuum, even though it is well known that the ice moves discontinuously, with fractures (leads) separating distinct rigid plates or floes. Thorndike [1986] recognized and discussed this problem. Using the satellite-derived ice motion data set of Hall and Rothrock [1981], he calculated the variance of velocity differences between points separated by 2 to $400 \mathrm{~km}$. He found powerlaw relationships between the variance and the separation distance, with exponents that implied the nonexistence of velocity derivatives in the limit of zero separation. He concluded that the ice velocity field has variations on all spatial scales. Thus a power-law scaling relationship is a natural way to describe sea ice deformation, and the nonexistence of velocity derivatives calls into question the basis of the continuum formulation of sea ice constitutive models. Recently Weiss et al. [2007, 2008] have analyzed simultaneous ice stress and strain rate measurements, finding that sea ice does not behave as a viscous material even at large scales. This was anticipated by Nye [1973], who speculated that there was no physical basis for assuming linear viscous behavior for sea ice. Apparently one cannot average over many deformation "events" and obtain fluid-like behavior. The alternative viewpoint of Weiss et al. is that fracture and frictional sliding govern inelastic deformation over all spatial and temporal scales, even under small stresses. The granular sea ice model of Hopkins and Thorndike [2006] supports this viewpoint.

[9] In the current work we extend Marsan et al. [2004] in two important ways: (1) We determine the spatial scaling of sea ice deformation rates over the course of seven years (November through July), obtaining a time series of the scaling exponent that shows a distinct seasonal cycle. The fact that sea ice deformation rates obey power-law scaling in all seasons lends validity to the viewpoint of Weiss et al. discussed above, and to the modeling approach of Hopkins and Thorndike [2006]. (2) We relate the scaling exponent to the fraction of multiyear ice, and discuss the implications in light of the decreasing amount of multiyear ice in the Arctic Ocean.

\section{Data}

[10] The data for this study come from the RADARSAT Geophysical Processor System (RGPS), which uses wideswath synthetic aperture radar (SAR) images acquired approximately every three days by the Canadian RADARSAT satellite as input to an ice-tracking algorithm that follows over 40,000 points on the sea ice by means of a crosscorrelation technique [Kwok et al., 1995; Kwok, 1998]. In November, after fall freezeup, the points are initialized on a regular square grid with $10 \mathrm{~km}$ spacing. They are tracked until the onset of melt in early summer, when the surface features in the SAR images become obscured. Points are then reinitialized and the new points are tracked through mid-August. The points define the corners of cells that are initially square but which drift and deform with the ice cover. The RGPS computes the ice velocities at these points from sequential SAR images, and then computes the sea ice deformation within each cell when the velocities of all four corners of the cell are observed over the same time interval. In particular, if $(x, y)$ represents position and $(u, v)$ represents velocity, the RGPS computes the four spatial velocity gradients or strain rates $(\partial u / \partial x$, etc.) using standard finite difference formulas, and the invariants of the strain rate tensor (divergence and shear rates, $\dot{D}$ and $\dot{S}$ ), for each cell. We refer to the total deformation rate as $\dot{\varepsilon}=\sqrt{\dot{D}^{2}+\dot{S}^{2}}$. Hereafter it is understood that deformation refers to the deformation rate $\dot{\varepsilon}$.

[11] The RGPS ice tracking is highly accurate, with error standard deviations on the order of 100 meters (one SAR image pixel). This leads to errors of about $0.5 \%$ day $^{-1}$ in $\dot{D}$ and $\dot{S}$ over a 3 -day period [Lindsay and Stern, 2003]. The RGPS also computes the area of multiyear ice within each cell, based on the SAR backscatter [Kwok et al., 1992]. The data are available from the RGPS web site. A recent issue of the Journal of Geophysical Research highlighted the use of RGPS data for studying sea ice kinematics and dynamics [Kwok and Coon, 2006].

[12] Due to the swath and orbit geometry of RADARSAT, the space/time sampling of the ice cover is irregular. We create "snapshots" of the sea ice deformation from the RGPS data as follows. We choose a target date/time, such as 00:00 UTC on 6 November 1997, and find all the RGPS deformation data that span the target time, i.e. for which the first observation is before the target time and the second observation is after the target time. This typically gives a spatially complete field of deformation, but different parts are computed over different time intervals, although they all span the same target time. We created 314 snapshots of sea ice deformation, with 5 days between snapshots, from November 1996 through May 2005. There are gaps, however: there is no data in late summer/early fall (August, September, October); no winter data in 2003 or 2004; and summer data only in 

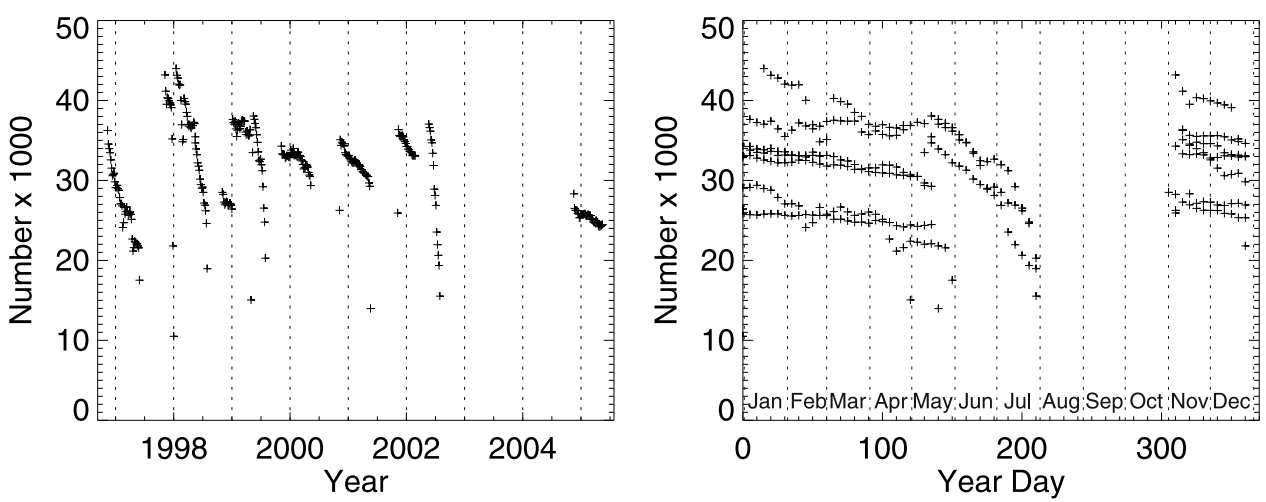

Figure 1. Number of cells in each snapshot (left) by year and (right) by day of the year (all years) for 297 snapshots.

1998, 1999, and 2002. Each snapshot contains tens of thousands of samples of sea ice deformation (one per cell). In the next section we describe filters that remove data that fall outside certain bounds, reducing the number of snapshots to 297 , with an average of 31,000 cells per snapshot. Figure 1 shows the number of cells per snapshot, distributed by year and by day of the year. Besides the deformation, data for each cell includes: observation time $t_{1}$ (before the nominal snapshot time), observation time $t_{2}$ (after the nominal snapshot time), time interval $\Delta t=t_{2}-t_{1}$, cell area at times $t_{1}$ and $t_{2}$, and multiyear ice area at times $t_{1}$ and $t_{2}$.

\section{Computing the Sea Ice Deformation Rate at Different Scales}

[13] A snapshot contains one sample of sea ice deformation per cell, which is on the order of $10 \mathrm{~km}$ across. We wish to construct estimates of sea ice deformation at larger spatial scales. We do this by aggregating cells, computing areaweighted averages of the strain rates $(\partial u / \partial x$, etc.), and computing the deformation invariants $(\dot{D}, \dot{S}, \dot{\varepsilon})$ from these averages, as in the work of Marsan et al. [2004]. The aggregation process is as follows. Choose a seed location $(X, Y)$ and a nominal length scale $W$. Find all the cells whose centers lie within $W / 2$ of $X$ in the $x$ direction and within $W / 2$ of $Y$ in the $y$ direction. Then apply the following constraints to each cell: (1) the time interval $\Delta t$ must be between 2 and 5 days; (2) the cell area at times $t_{1}$ and $t_{2}$ must be between 50 and $200 \mathrm{~km}^{2}$; (3) the total deformation rate $\dot{\varepsilon}$ must be less than $100 \%$ day $^{-1}$. The constraint (1) ensures that the deformation rates in different cells span similar time intervals. The constraints (2) and (3) attempt to filter out possibly erroneous data based on unreasonable area changes or cell deformation, which might arise due to errors in the ice tracking, or due to the extreme sensitivity of the deformation estimates in highly distorted cells. These constraints must attempt to strike a balance between allowing real extreme events and excluding erroneous extreme events. Finally, after cells are eliminated based on the constraints, the total area of the retained cells must be greater than $0.75 W^{2}$ (i.e., $75 \%$ of the nominal $W \times$ $W$ box). If this final condition is met, then the area-weighted averages of $\partial u / \partial x$ and the other strain rates are computed for the collection of retained cells, and the deformation invariants $(\dot{D}, \dot{S}, \dot{\varepsilon})$ are computed from the averages. Thus we obtain one estimate of the deformation at the nominal scale $W$.
[14] For the actual spatial scale associated with the deformation, we use the square root of the total area of the retained cells, which we denote by $L$. The total area $L^{2}$ may be greater than the nominal area $W^{2}$ because only the cell centers are required to be within the nominal $W \times W$ box.

[15] The seed point $(X, Y)$ is then shifted in the $x$ or $y$ direction by $W / 2$ (if $\mathrm{W}>100 \mathrm{~km}$ ) or by $50 \mathrm{~km}$ (if $\mathrm{W}<$ $100 \mathrm{~km}$ ), and the aggregation process is repeated. In this way, many estimates of $\dot{\varepsilon}$ at the nominal scale $W$ are computed. Note that these estimates are not independent (for $\mathrm{W}>50 \mathrm{~km}$ ) because adjacent $W \times W$ boxes overlap.

[16] The entire procedure above is repeated for different values of $W$ to get estimates of $\dot{\varepsilon}$ at different spatial scales. We use 10 values of $W$ logarithmically spaced between 10 and $1000 \mathrm{~km}$. At the smallest scale, no aggregation of cells is necessary; we simply select a subsample of all the cells in the snapshot. Figure 2 shows the spatial distribution of the $10-\mathrm{km}, 360-\mathrm{km}$, and $1000-\mathrm{km}$ samples for a single snapshot.

\section{Spatial Scaling of the Mean Deformation Rate}

[17] The result of the procedure described in the previous section is 297 sets of deformation estimates and their associated spatial scales, $\dot{\varepsilon}$ and $L$, one set for each snapshot. Figure 3 is a plot of $\dot{\varepsilon}$ versus $L$ for the snapshot of 20 April 1999. The data cluster around the nominal scales, which are separated by vertical dashed lines, and these define 11 bins. Let $\langle\dot{\varepsilon}\rangle_{k}$ and $\langle L\rangle_{k}$ denote the mean deformation and mean scale for bin $k(k=1$ to 11$)$. In forming the bin averages we require at least 10 samples per bin, which occasionally reduces the maximum scale included in the analysis. We postulate a power-law relationship of the form $\langle\dot{\varepsilon}\rangle=c\langle L\rangle^{b}$ and apply a standard least squares fitting procedure in log-log space to determine the two constants by minimizing the variance of the error term $E$ :

$$
\log \langle\dot{\varepsilon}\rangle_{k}=a+b \log \langle L\rangle_{k}+E_{k} \quad(k=1 \text { to } 11)
$$

We apply this fitting procedure to each snapshot, obtaining time series of the scaling parameters $a$ and $b$. The squared correlations of the fits in equation (1) are quite high (mean 0.96), with 279 exceeding 0.90 . This indicates that the power law is a good model for relating the mean deformation to the spatial scale. 


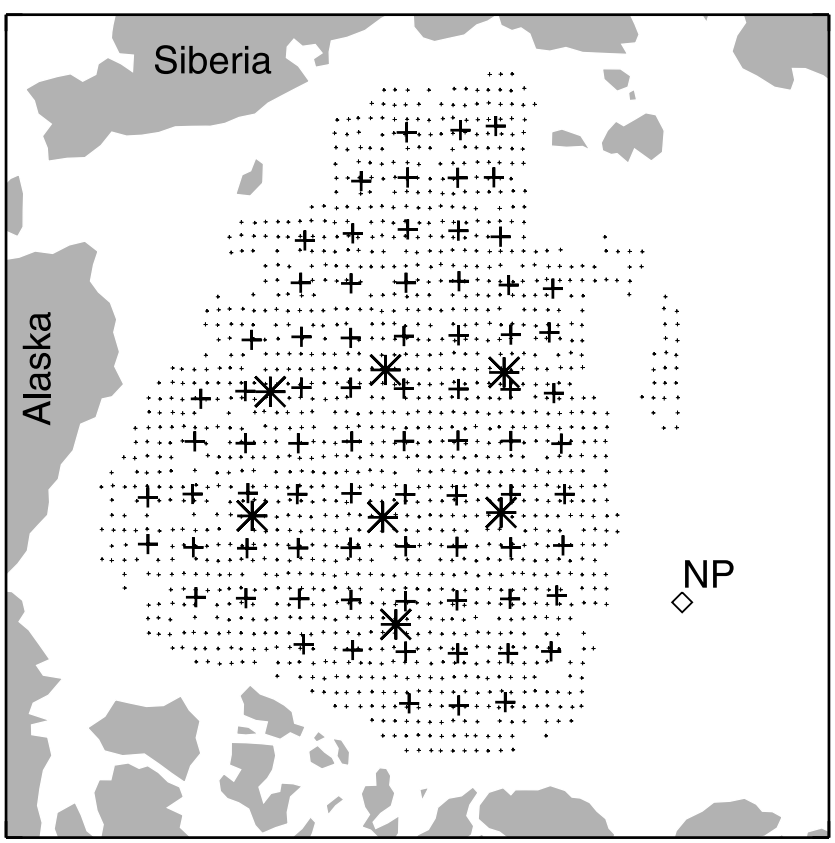

Figure 2. Grid sampling of the snapshot from 20 April 1999 showing the center positions of all the samples for the $10-\mathrm{km}$ scale (dots), the $360-\mathrm{km}$ scale (crosses), and the $1000-\mathrm{km}$ scale (stars). The 10-km samples are separated by $50 \mathrm{~km} . \mathrm{NP}$ is the North Pole.

[18] The power-law scaling arises from the spatial structure of the deformation field, not from the sampling procedure. We verified this (as in the work of Marsan et al. [2004]) by a Monte Carlo simulation of 100 random rearrangements of the deformation field. In each rearrangement or shuffling, all the deformation values $(\dot{\varepsilon})$ of the RGPS cells (for a given snapshot in time) were randomly assigned to the locations of

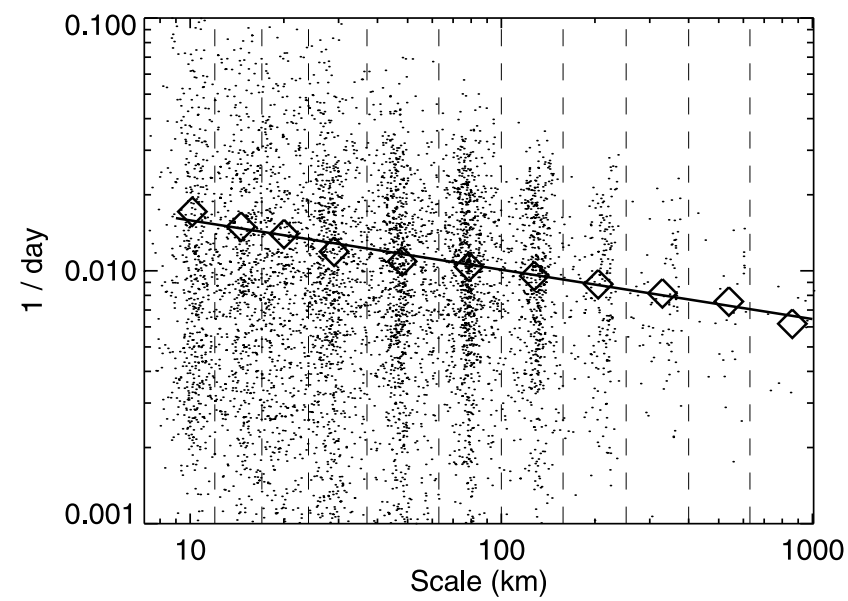

Figure 3. Total deformation $\dot{\varepsilon}\left(\right.$ day $\left.^{-1}\right)$ versus spatial scale $L(\mathrm{~km})$ for the 6778 samples of deformation computed from the snapshot of 20 April 1999. Each dot represents one estimate of the deformation (vertical axis) at a particular spatial scale (horizontal axis). The vertical dashed lines delineate the scale bins. The diamonds show the mean deformation for each bin, and the straight line is the best linear fit to the means, with a slope of $b=-0.15$ and a squared correlation of 0.96 .

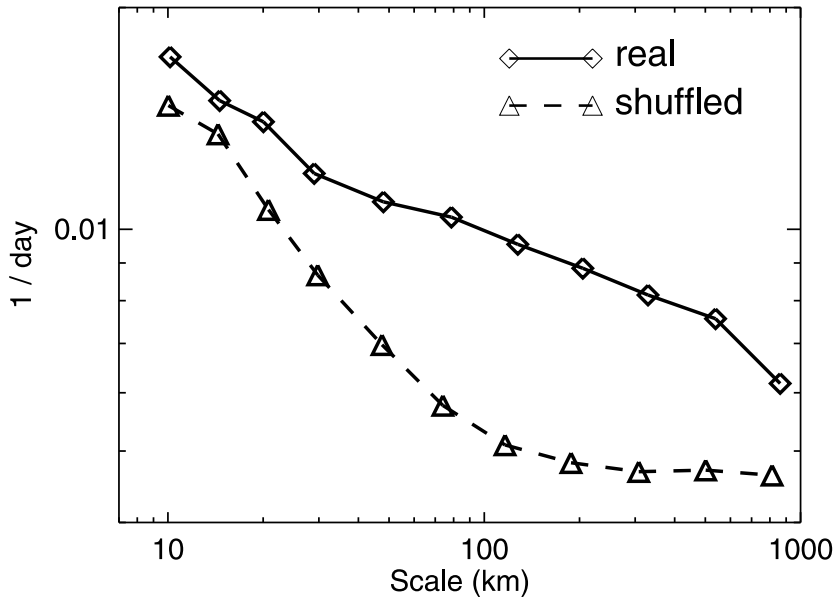

Figure 4. Mean deformation $\dot{\varepsilon}\left(\right.$ day $\left.^{-1}\right)$ versus spatial scale $L(\mathrm{~km})$ for the snapshot of 20 April 1999, showing the actual mean values (upper curve, same as the diamonds in Figure 3), and those resulting from a random shuffling of the deformation field prior to the scaling analysis (lower curve, triangles). The clear departure from linearity in the lower curve indicates that the spatial structure of the deformation field is responsible for the observed power-law scaling (approximate linearity) in the upper curve.

other cells, thus destroying the spatial patterns in the deformation field while maintaining the same set of deformation values and the same cell locations. For each shuffled field we then repeated the scaling analysis described in section 3 and applied the fitting procedure of equation (1). We always found a clear departure from power-law behavior in $\langle\dot{\varepsilon}\rangle$ versus $\langle L\rangle$ for the shuffled fields. Figure 4 shows an example from the snapshot of 20 April 1999. The actual (unshuffled) data give rise to a nearly linear relationship between $\langle\dot{\varepsilon}\rangle$ and $\langle L\rangle$ in log-log space (upper curve, same as the diamonds in Figure 3), whereas the shuffled data do not (lower curve). Hence it is the spatial arrangement of the actual (unshuffled) deformation values that leads to the power-law scaling.

[19] Figure 3 also shows that the variance of the deformation about the mean is large. Since the deformation tends to concentrate along active fault lines (leads) in the ice cover, a set of deformation estimates at a given spatial scale will tend to include many small values (from rigid pieces that do not deform) and many large values (from the active zones), giving rise to a broad distribution of values. The variance increases as the spatial scale decreases (as also noted by Marsan et al. [2004]), which is related to the localization discussed in section 6 .

\section{Annual Cycle of Scaling Parameters}

[20] Figure 5 shows the scaling exponent $b$ by year and by day of the year. The exponent has a mean value of -0.18 (standard deviation 0.04) during November through April, and then increases in magnitude during the melt season, reaching about -0.25 by August when our data end. The increasing summer exponent is consistent with a looser ice pack in which stresses are not transmitted long distances, so the connection between distant parts of the ice pack is weaker. After freezeup in the fall, the exponent reverts to its winter 

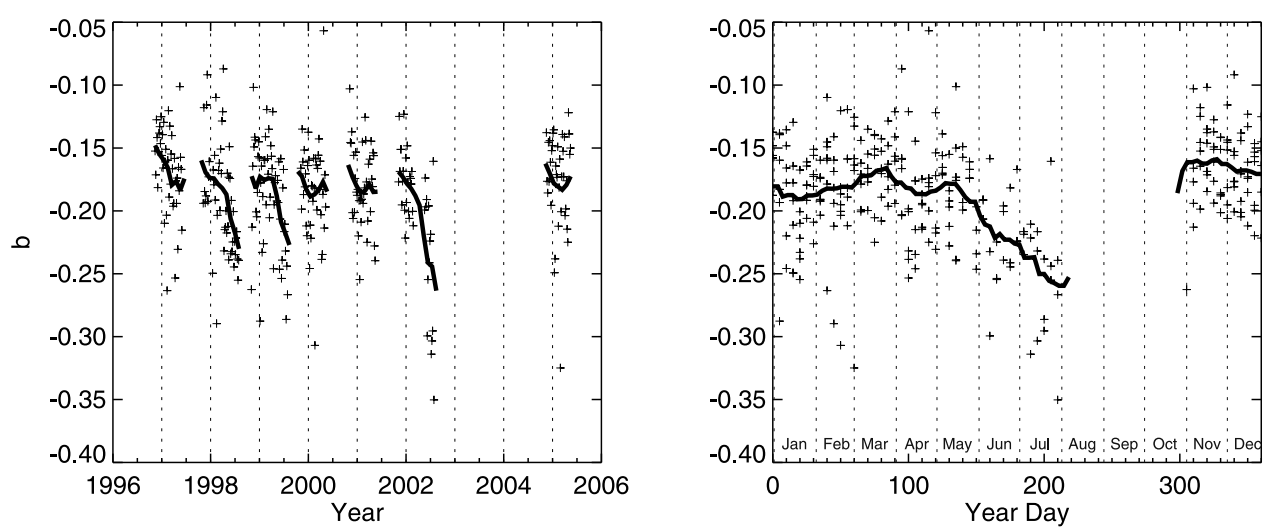

Figure 5. Power-law scaling exponent (left) by year and (right) by day of the year (all years) for 297 snapshots (crosses). The curves on the left are 4-month running means and those on the right are 1-month running means.

value. The mean value over all months (November through July) of -0.20 confirms that the exponent obtained by Marsan et al. [2004] is typical. The annual cycle in Figure $5 \mathrm{~b}$ agrees with that obtained from RGPS data at scales from $5 \mathrm{~km}$ to $200 \mathrm{~km}$ centered on the SHEBA station in the Beaufort Sea in 1997-1998 [Stern and Moritz, 2002]. The scaling exponents from that data set have a mean value of $-0.18(n=68$, standard deviation 0.10$)$ during November through April, and a mean value of -0.40 in August $(n=9$, standard deviation 0.10 ), showing a clear separation between winter (frozen) conditions and summer (free drift) conditions.

[21] Why is the winter scaling exponent on the order of -0.2 and not (say) ten times larger or smaller? The answer is largely a reflection of the strength of sea ice. If the ice were much weaker, the exponent would be more negative; if the ice were much stronger, the exponent would be closer to zero. Alternatively, one could cast it in terms of the external forcing: if the wind stress gradients were much larger, the exponent would be more negative; if the wind stress gradients were much smaller, the exponent would be closer to zero. The particular value -0.2 simply reflects the balance between the external forcing and the internal response of the ice cover.

[22] Figure 6 shows the constant of proportionality from the scaling relationship, $c=\exp (a)$, by year and by day of the year. It can be interpreted as the mean deformation rate at a scale of $1 \mathrm{~km}$, as extrapolated from the best fit solution to equation (1). The distinct annual cycle is clear, with a minimum in March when the pack ice is nearly locked up, and increasing values in summer as the pack ice loosens up. The annual cycle of the mean deformation at larger scales exhibits similar patterns, but scaled down by a factor of $L^{b}$ where $L$ is the spatial scale and $b$ is the exponent shown in Figure 5. For example, the mean deformation in March at the $1-\mathrm{km}$ scale is about $0.02 \mathrm{day}^{-1}$ (Figure $6 \mathrm{~b}$ ), so at the $10-\mathrm{km}$ scale it is about $0.02 \times 10^{-0.16}=0.014 \mathrm{day}^{-1}$. This generally agrees with the March sea ice deformation obtained by Kwok [2006] for the years 1997-2000 using RGPS data at the $10-\mathrm{km}$ scale. He also found that the deformation reached a minimum in March, with higher values in the spring and fall. Our results show that the deformation continues to increase right into the summer (based on the years with summer data: 1998, 1999, 2002), probably reaching a maximum in August before reversing with the start of fall freezeup in September. The RGPS data centered on the SHEBA station in summer 1998 [Stern and Moritz, 2002] give mean values of $c$ (deformation extrapolated to $1-\mathrm{km}$ scale) of 0.036 day $^{-1}$ in June and 0.054 day $^{-1}$ in July, in agreement with Figure 6b, but in August the mean shoots up to $0.26 \mathrm{day}^{-1}$ following the general break-up of the ice pack in the vicinity of the SHEBA station at the end of July 1998. The spike in deformation
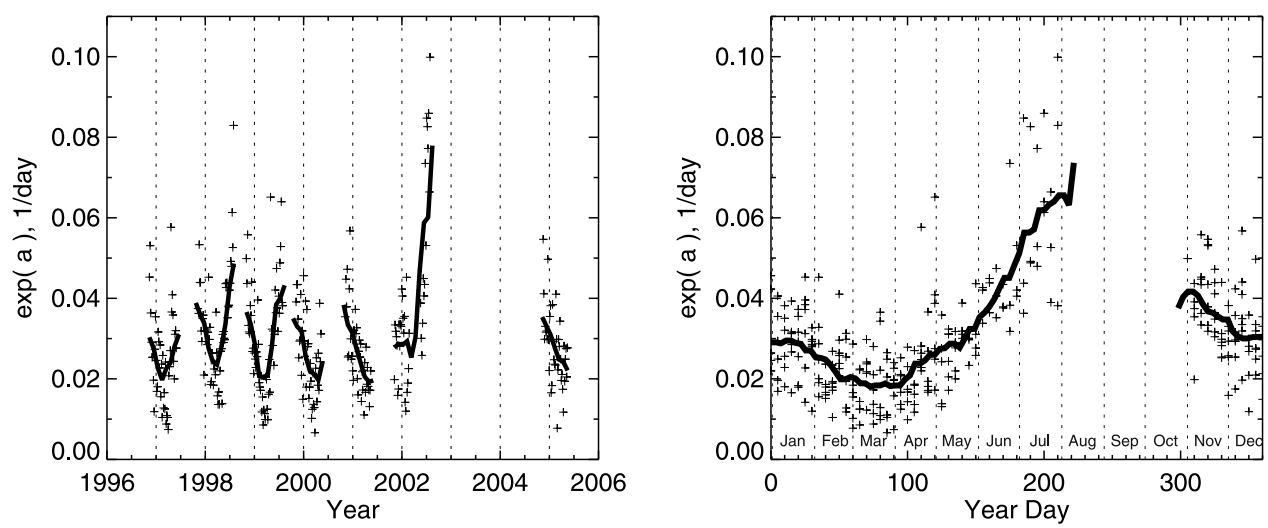

Figure 6. Deformation at 1-km scale extrapolated from least squares fits, (left) by year and (right) by day of the year (all years) for 297 snapshots (crosses). The curves on the left are 4-month running means and those on the right are 1-month running means. 


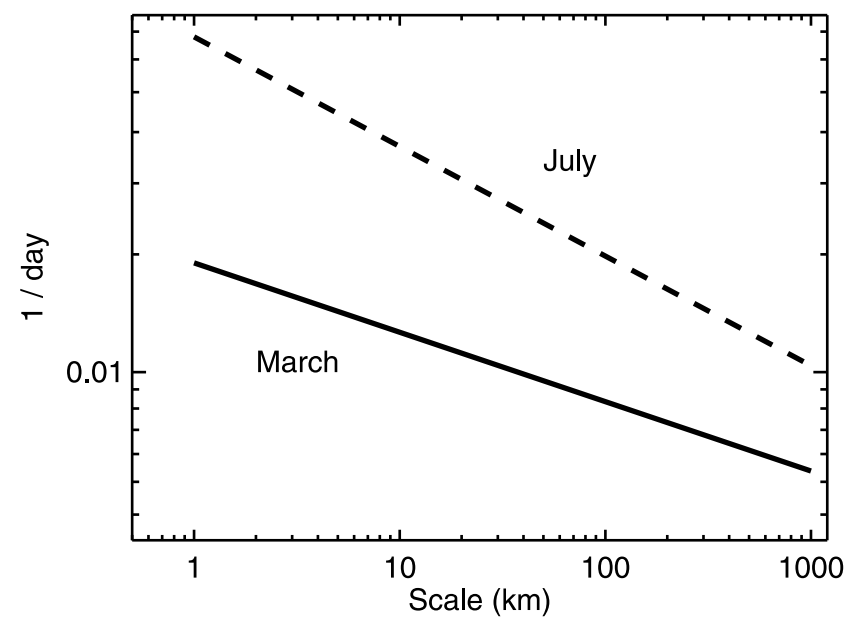

Figure 7. Best fit lines for the mean deformation versus scale in March (all years, 24 snapshots, $b=-0.16$ ) and in July (all years, 18 snapshots, $b=-0.26$ ).

in July 2002 (Figure 6a) is probably due to an early break-up and transition to free-drift conditions, with typical values of August deformation occurring in July instead. We note that Arctic sea ice extent in August 2002 was a record low for all Augusts since 1979 (based on satellite passive microwave data), perhaps facilitated by the early break-up in July.

[23] To contrast the scaling relationships in winter and summer, Figure 7 shows the best fit lines to the mean deformation versus scale for all March data and all July data. The mean deformation in summer is greater at all scales, and it increases more rapidly with decreasing scale. This is because the summer sea ice cover is thinner, weaker, and less compact, with less internal stress, which may allow the spatial variability in the wind and water stress fields to have a greater effect. Also, in summer the internal stresses cannot be transmitted long distances, and the coastal boundary cannot exert its influence far offshore, so the deformation must decrease more steeply as the scale increases. This leads to a negative correlation $(\mathrm{R}=-0.57, \mathrm{~N}=40)$ between the scaling exponent $b$ and the constant of proportionality $c=\exp (a)$. In winter, however, there is no correlation $(\mathrm{R}=0.04, \mathrm{~N}=231)$. In other words, the rate at which the deformation increases with decreasing scale is not related to the magnitude of the small-scale deformation; the two parameters are independent, and both are necessary to characterize the deformation.

[24] The higher deformation rates in summer are not due to stronger winds. Wind speeds in summer and winter over the Arctic Ocean are about the same [Lindsay, 1998], and cyclone counts are about the same [Zhang et al., 2004]. Summer cyclones are actually less intense than those in winter, although they tend to last slightly longer [Zhang et al., 2004].

[25] Kwok [2006] attributed the seasonal trend toward the deformation-minimum in March to three factors: increasing ice thickness and hence mechanical strength; the effect of coastal boundaries after the basin becomes ice-filled in early winter; and reduced momentum transfer from the atmosphere to the ice in winter due to a more stable atmospheric boundary layer. He also found higher deformation rates in the seasonal ice zone (SIZ) than in the perennial ice zone (PIZ) in all months (October to May), as we do (see section 7).
[26] Stern and Moritz [2002] distinguished the "thermodynamic" summer (beginning with melt onset) from the "kinematic" summer (beginning with break-up), based on data from the SHEBA station during 1997-1998. Melt onset at SHEBA began at the end of May, but the ice remained relatively rigid until the end of July, when a storm finally broke up the weakened ice pack. This transition to free-drift conditions was also marked by a sudden change in the scaling exponent, which became more negative. A sharp summer transition is not evident in Figures $5 \mathrm{a}$ or $5 \mathrm{~b}$, however, because the scaling exponent is calculated from data over the whole domain of Figure 2, rather than regionally. In section 7 we look at regional patterns.

\section{Localization}

[27] The "localization" of deformation means that as the scale of measurement decreases, the largest deformation rates are confined to smaller and smaller areas. Suppose for a given snapshot in time and a given spatial scale $L$, the deformation estimates are arranged from largest to smallest: $\dot{\varepsilon}_{1}>\dot{\varepsilon}_{2}>\ldots>$ $\dot{\varepsilon}_{n}$. The mean deformation is $\langle\dot{\varepsilon}\rangle=\left(\dot{\varepsilon}_{1}+\dot{\varepsilon}_{2}+\ldots+\dot{\varepsilon}_{n}\right) / n$. Let $k$ be the smallest integer such that $\left(\dot{\varepsilon}_{1}+\dot{\varepsilon}_{2}+\ldots+\dot{\varepsilon}_{k}\right) / n \geq$ $0.15\langle\dot{\varepsilon}\rangle$. Then the area $A_{1}+A_{2}+\ldots A_{k}$ associated with the deformation estimates $\dot{\varepsilon}_{1}$ through $\dot{\varepsilon}_{k}$ is said to accommodate the largest $15 \%$ of the deformation. Marsan et al. [2004] examined the largest $15 \%$ of the deformation at each scale (for a single snapshot in time), and found that the fractional area accommodating this deformation decreased from $3.2 \%$ to $1.6 \%$ as the scale decreased from $200 \mathrm{~km}$ to $20 \mathrm{~km}$. Following the same idea, we computed the area fraction containing the largest $15 \%$ of the deformation for each scale range, for all 297 snapshots in time. The area fraction does indeed decrease as the scale decreases (Figure 8, solid curve). When we randomly shuffled the deformation values of the RGPS cells among themselves, as in section 4 for the scaling analysis, we found the localization to be much weaker (Figure 8, dashed curve): the area accommodating the largest $15 \%$ of the deformation is much larger (less localized) for the shuffled data than for the original, unshuffled data (at scales

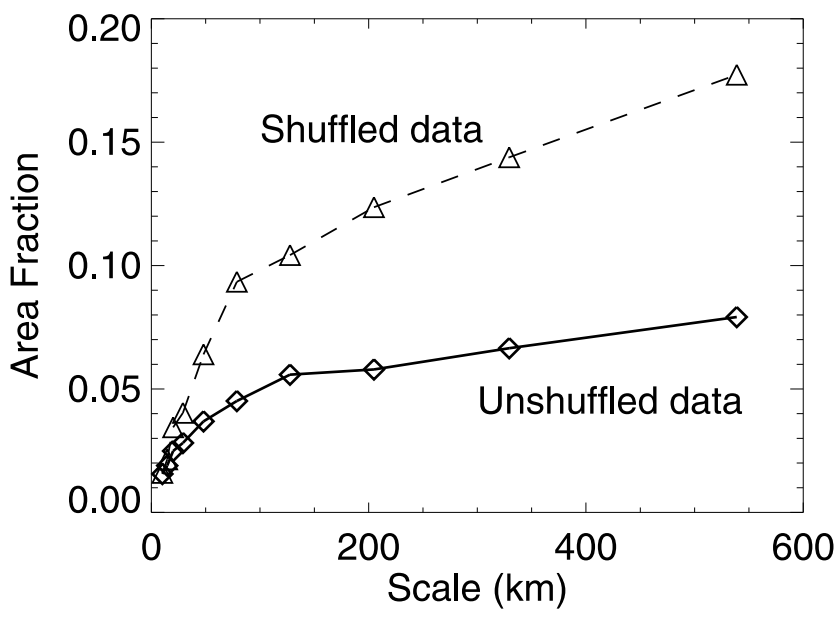

Figure 8. Area fraction accommodating the largest $15 \%$ of the deformation, as a function of spatial scale, for shuffled (dashed) and unshuffled (solid) data from the snapshot of 20 April 1999. 


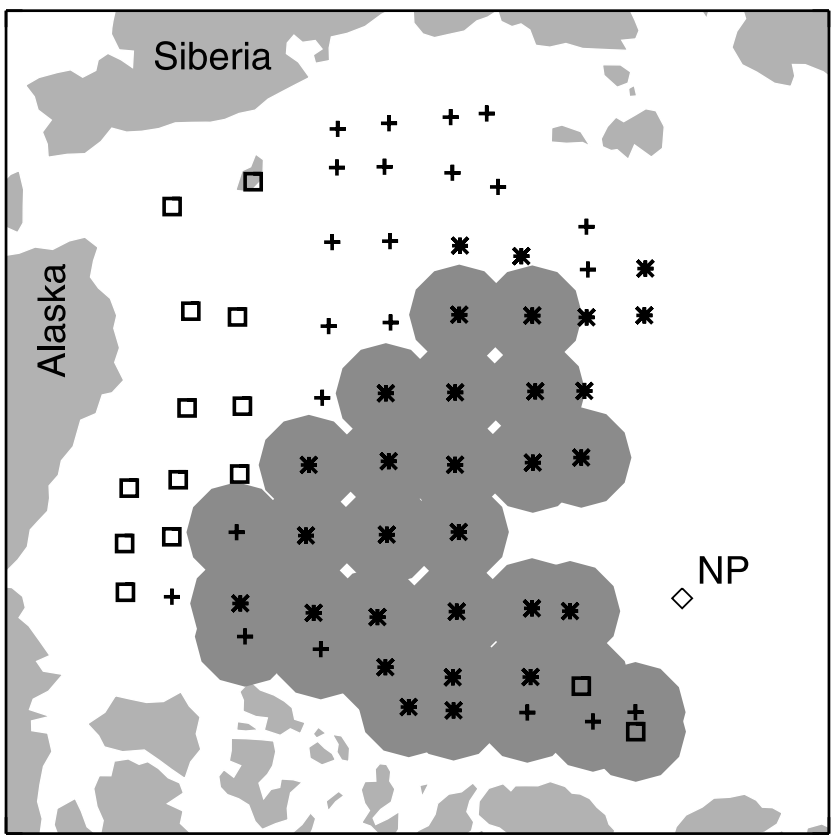

Figure 9. Scaling exponents $b$ (symbols) and multiyear ice $>80 \%$ (gray) for pooled data from November 1997 through January 1998. Scaling exponents: $(*) 0.00$ to $-0.15 ;(+)-0.15$ to $-0.20 ;(\square)-0.20$ to -0.40 . NP is the North Pole.

larger than $50 \mathrm{~km}$ ). Note that the area fraction is necessarily the same at the $10-\mathrm{km}$ scale for both the shuffled and unshuffled data, since that is the scale of the cells that were shuffled. Thus we find much stronger localization (smaller area accommodating the largest deformation) in the actual deformation field than in the spatially shuffled field, indicating that the high degree of localization is a property of the spatial structure of the field. The localization persists through all the snapshots with no appreciable annual cycle.

[28] A homogeneous deformation field would have the largest $15 \%$ of the deformation accommodated by $15 \%$ of the area. As shown in Figure 8, the actual area (solid curve, unshuffled data) remains well below $15 \%$ at all scales, indicating that the deformation field is highly heterogeneous whatever the scale considered.

[29] In addition to occupying a smaller and smaller area as the scale decreases, the largest $15 \%$ of the deformation also increases in magnitude at a faster rate than the mean deformation shown in Figure 3. Thus the localization also includes a strengthening of the largest deformation events at smaller scales. These localization properties suggest that much of the deformation is brittle, being accommodated by differential displacements along fracture and fault planes, and is best described using fracture mechanics rather than continuum mechanics [Weiss et al., 2008].

\section{Regional Patterns and Multiyear Ice}

[30] Each least squares fit of equation (1) uses estimates of $\dot{\varepsilon}$ and $L$ obtained from one snapshot, a single point in time, distributed over the spatial domain shown in Figure 2. Alternatively, to investigate regional patterns, we pool estimates of $\dot{\varepsilon}$ and $L$ from all the winter snapshots, but restrict each analysis to a $500 \times 500 \mathrm{~km}$ subregion of the whole domain. Then we do the scaling analysis (least squares fit) of equation (1), shift the $500 \times 500 \mathrm{~km}$ box by $250 \mathrm{~km}$, and repeat the procedure across the whole domain. For each least squares fit we use 10 scales (bins) from 10 to $500 \mathrm{~km}$, but if a bin has fewer than 10 samples we discard it, so not every bin is used in every fit.

[31] Figure 9 is a map of the power-law scaling exponents $b$ obtained by this procedure, using snapshots from November 1997 through January 1998. The exponents are on the order of -0.15 in the central Arctic, and -0.20 or less in a broad band along the coasts of Alaska and Siberia. The exponents, with mean value -0.18 (standard deviation $0.08, N=66$ ), are very similar to those obtained from single snapshots during the same period (see Figure 5). The gray shading is where the multiyear (MY) ice fraction is greater than $80 \%$ within the region centered on each symbol over the 3-month time period. (This is based on the RGPS MY ice area for each $10-\mathrm{km}$ cell, which is derived from the SAR backscatter [Kwok et al., 1992]). It is evident that the scaling exponents tend to be smaller in magnitude where the MY ice fraction is high. MY ice tends to be thicker and stronger than first-year (FY) ice, allowing internal stresses to be transmitted over longer distances, so the deformation versus scale has a shallower slope (i.e., there is a stronger connection between distant parts of the ice pack). In contrast, the band along the coasts of Alaska and Siberia has more FY ice, which tends to be thinner; it behaves more like a summer ice pack in which stresses are not transmitted long distances, so the deformation versus scale has a steeper slope (weaker connection between distant points), hence the exponents are larger in magnitude there. The same general pattern is found in other 3-month winter maps.

[32] Figure 10 is the corresponding map showing the constant of proportionality from the regional power-law

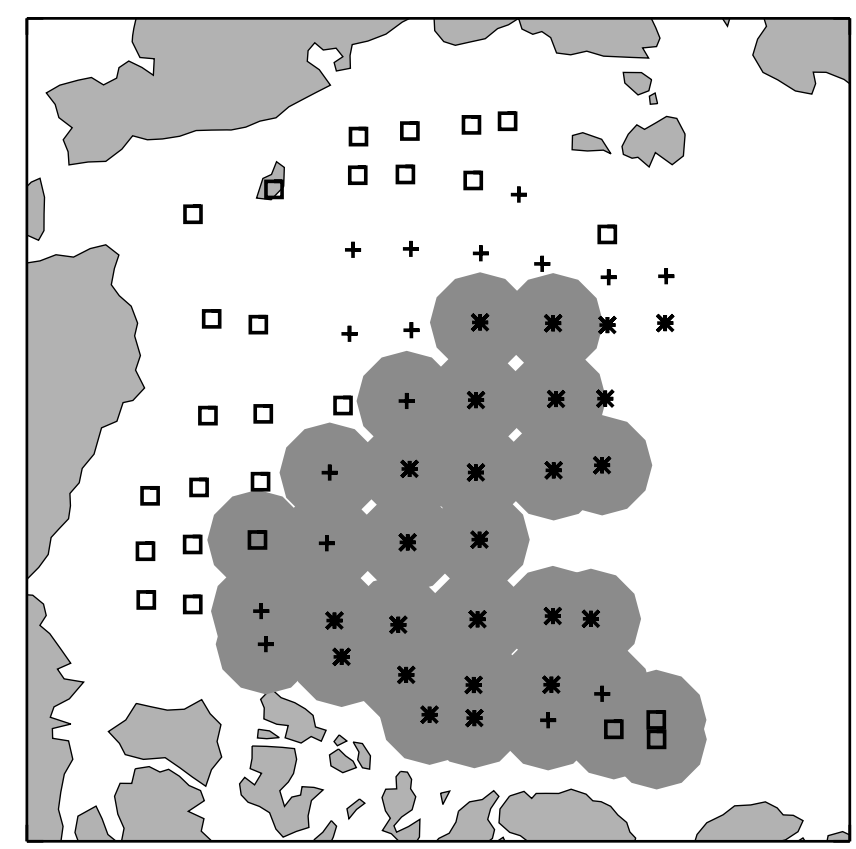

Figure 10. Scaling constants $c$ (symbols) and multiyear ice $>80 \%$ (gray) for pooled data from November 1997 through January 1998 . Scaling constants: $(*) 0$ to 0.026 day $^{-1}$; (+) 0.026 to $0.040 \mathrm{day}^{-1}$; (口) 0.040 to $0.100 \mathrm{day}^{-1}$. 

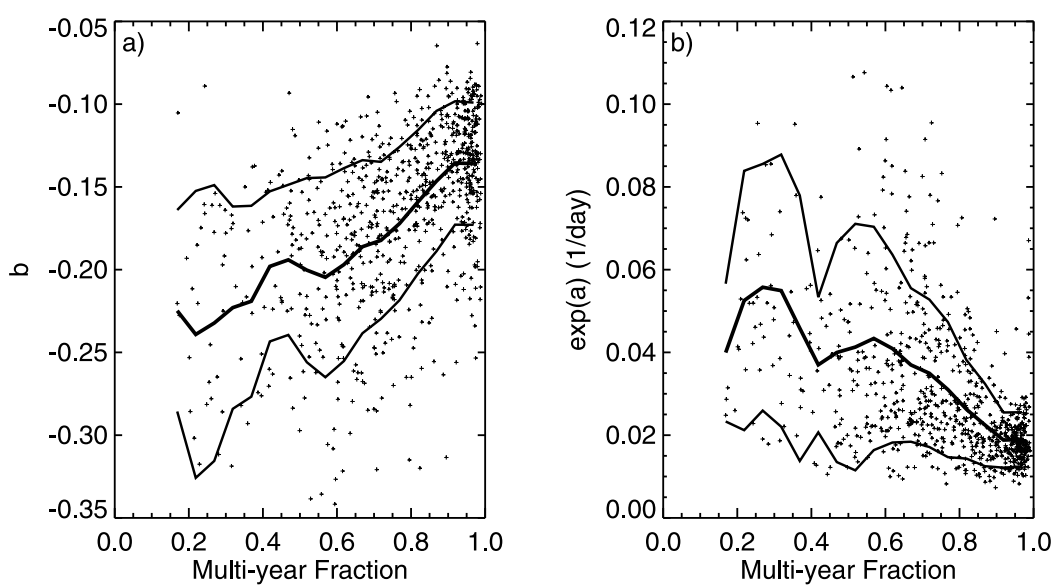

Figure 11. (left) The power-law exponents $b$ and (right) the constants of proportionality $c=\exp (a)$ versus the multiyear ice fraction for distributions of the total deformation within 500-km boxes and 3-month winter periods (from all years). The thick curves are running means using a window width of 0.1 in multiyear ice fraction, and the thin curves are \pm 1 standard deviation.

fits, $c=\exp (a)$, which is also the deformation at the $1-\mathrm{km}$ scale as extrapolated using equation (1). Regions of low deformation (* symbol) correspond with high MY ice fractions (gray shading). Similar patterns are found in the other 3-month winter maps. The pattern is essentially the same as that found by Kwok [2006], who also used RGPS data to look at sea ice deformation and MY ice concentration. The shaded regions in Figures 9 and 10 use the same MY ice threshold of $80 \%$ as Kwok's perennial ice zone (PIZ). He found that the deformation is two to three times larger in the seasonal ice zone ( $\leq 80 \% \mathrm{MY}$ ice) than in the PIZ, which can also be seen in Figure 10. Our interest is mainly in the scaling of deformation (exponent $b$ ), but this confirms that the spatial patterns of the magnitude of deformation (parameter $c$ ) and the MY ice fraction agree with those obtained by Kwok [2006].

[33] Figure 11 shows the power-law scaling exponents $b$, and the constants of proportionality $c=\exp (a)$, both versus MY ice fraction. Each data point represents a $500 \times 500 \mathrm{~km}$ region and a 3-month winter period (either NovemberJanuary or February-April, all years; $\mathrm{N}=911$ data points). The thick curves are running means, and the thin curves are \pm 1 standard deviation. The scaling exponents (a) decrease in magnitude from $b=-0.24$ for MY fractions of about 0.2 , to $b=-0.14$ for MY fractions near 1 . The small-scale deformation (b) is smaller and exhibits much less variability for large multiyear fractions, decreasing from $\exp (a)=$ 0.05 day $^{-1}$ at MY fractions of 0.2 to $\exp (a)=0.02$ day $^{-1}$ at MY fractions near 1. Multiyear ice tends to be thicker and stronger than first-year ice, accounting for both the reduced magnitude and the reduced variability of the small-scale deformation, since stronger ice simply does not allow frequent, large deformation events. Figures 9 and 10 are spatial illustrations of these MY ice tendencies for a single 3-month period. Another perspective is given in Figure 12, showing the best fit lines for the mean deformation versus scale for regions with high and low MY ice fractions. The mean deformation is greater at all scales for low MY ice regions, and it increases more rapidly with decreasing scale. Compare this with Figure 7: low MY ice regions behave like a summer ice pack, and high MY ice regions behave like a winter ice pack.

\section{Discussion and Conclusions}

[34] We have shown that the mean deformation rate of Arctic sea ice depends on the spatial scale over which it is measured according to a power-law relationship of the form $\langle\dot{\varepsilon}\rangle=c\langle L\rangle^{b}$ where the exponent $b$ is about -0.2 in winter and larger (in magnitude) in summer. The size of the scaling exponent determines how steeply the mean deformation increases as the scale decreases. For a winter exponent of -0.2 , a doubling of the mean deformation occurs for a decrease in scale by a factor of 30 ; for a summer exponent of -0.3 , a doubling of the mean deformation occurs for a decrease in scale by a factor of only 10 . This is because the looser summer ice pack does not allow internal stresses to

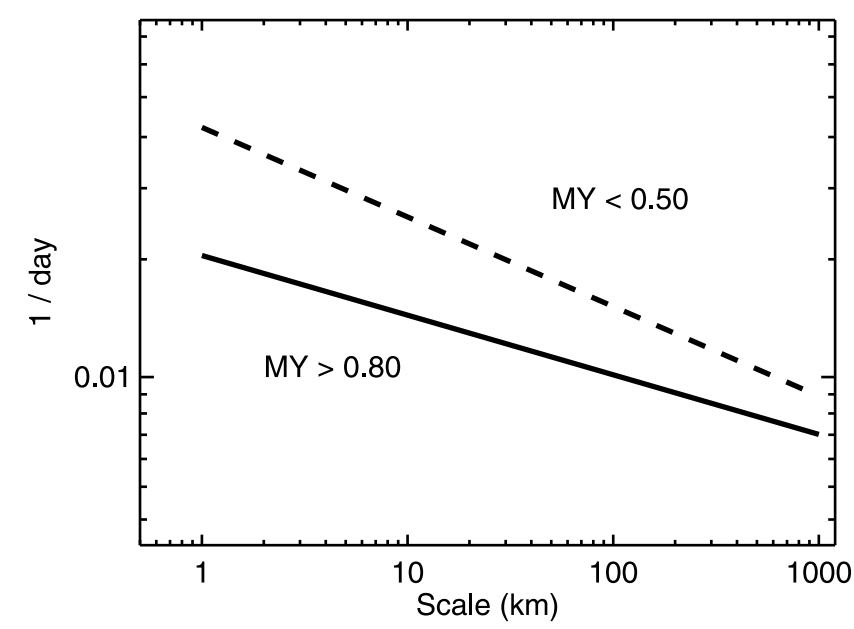

Figure 12. Best fit lines for the mean deformation versus scale for $\mathrm{MY}<0.50(b=-0.21, \mathrm{~N}=97)$ and for $\mathrm{MY}>0.80$ $(b=-0.14, \mathrm{~N}=403)$. 
be transmitted long distances, the influence of the coast is lost when the ice melts back from shore, and the ice is less constrained as the scale decreases, hence freer to respond directly to the wind-forcing. The fact that the mean deformation obeys power-law scaling indicates that there is no preferred or characteristic scale to the deformation process, within the scale range examined here (10 to $1000 \mathrm{~km})$.

[35] Scaling relationships are useful for comparing measurements made at different scales. Sea ice deformation can be computed from spatial fields of ice motion based on satellite data (e.g., SAR, AVHRR, SSM/I) or model output, typically all with different grid sizes. Suppose the deformation is computed from source $A$ on a $25-\mathrm{km}$ grid and from source $B$ on a $75-\mathrm{km}$ grid, and we wish to compare the deformation over a $300 \times 300 \mathrm{~km}$ region. Source $A$ provides 144 independent measurements of the $25-\mathrm{km}$ deformation, from which we compute a mean deformation $\langle\dot{\varepsilon}\rangle_{A}$; source $B$ provides 16 measurements of the $75-\mathrm{km}$ deformation, from which we compute a mean deformation $\langle\dot{\varepsilon}\rangle_{B}$. We then expect $\langle\dot{\varepsilon}\rangle_{A} /\langle\dot{\varepsilon}\rangle_{B}=(25 / 75)^{-0.2}=1.25$ (assuming a typical winter scaling exponent of -0.2 ), i.e. we expect the mean deformation from source $A$ to be $25 \%$ larger than from source $B$, due solely to the scaling effect. This is an important correction in model-model and model-data comparisons. (A first attempt to account for this scaling effect in model-data comparisons was made by Lindsay et al. [2003]). The existence of the scaling property also suggests a new validation metric for sea ice models: from the model velocity field, compute estimates of sea ice deformation on a range of spatial scales (as in section 3), and check to see whether or not the mean deformation versus scale follows a power law. This has recently been done by Girard et al. [2009] for two sea ice models, neither of which reproduced the observed scaling properties, possibly suggesting a problem in the mechanical framework of the models.

[36] Another property of the sea ice deformation field is localization: the largest $15 \%$ of the deformation is accommodated by a smaller portion of the ice cover as the scale decreases, and involves larger deformation rates. Hopkins and Thorndike [2006] observed this property in their granular model of sea ice, which consists of thousands of individual grains of size $\sim 10 \mathrm{~km}$ that can freeze together, fracture apart, and ridge. The simulation of these processes leads to the localization of deformation and a power-law floe size distribution, highlighting the importance of fracturing in recreating the observed structure of pack ice. While this argues for sea ice models based on fracture mechanics or granular interactions, continuum sea ice models are currently more computationally efficient and can be coupled more naturally to continuum ocean and atmosphere models.

[37] It is well known that Arctic sea ice extent and thickness are decreasing [Parkinson and Cavalieri, 2008; Meier et al., 2007; Lindsay et al., 2009; Kwok et al., 2009], and that younger, thinner ice is replacing thicker perennial ice [Haas et al., 2008; Maslanik et al., 2007; Nghiem et al., 2007]. According to the results in section 7, this decrease in MY ice should lead to larger deformation rates and to a steeper slope in the mean deformation versus spatial scale, as in Figure 12. In fact, larger deformation rates have already been observed [Rampal et al., 2009]. How does this feed back to the sea ice thickness? Larger deformation implies enhanced ice production in winter (from divergence) and ridging (from convergence). Ridging creates thicker ice, a negative feedback on the sea ice thickness distribution. There are also several positive feedbacks. In summer, a more divergent ice pack exposes more open water to solar heating, leading to more melting and thinner ice, the positive icealbedo feedback. A thinner ice pack has already led to higher ice drift speeds [Rampal et al., 2007, 2009] which may transport more ice out of the Arctic, leaving behind a younger and thinner ice pack, a positive dynamic feedback. Kwok [2006] noted that enhanced winter ice production from increased divergence leads to increased brine rejection, which could reduce the stability of the water column, allowing more ocean heat to reach the underside of the ice; and shearing motions of the ice cover may promote upwelling that would also bring warmer water to the surface, melting more ice. The strengths of these positive feedbacks have not been quantified, although reductions in sea ice extent and thickness are projected to continue [IPCC, 2007], undoubtedly accompanied by an increase in sea ice deformation rates.

[38] Returning to the scaling relation, in which the scaling exponent $b$ becomes more negative as MY ice decreases, this implies a weakening of the connection between regions of the ice cover that are separated by large distances. This does not necessarily trigger feedbacks to the sea ice deformation or thickness, but rather is an indicator of reduced ice strength.

[39] Acknowledgments. This work was supported by the National Science Foundation (ARC-0612023), including a separate travel grant (INT-0128689). We thank Jérôme Weiss, David Marsan, and Pierre Rampal for many helpful discussions and two anonymous reviewers for their constructive comments. The RGPS data are provided by Ron Kwok and the RGPS team at the Jet Propulsion Laboratory, Pasadena, CA, with funding from NASA.

\section{References}

Girard, L., J. Weiss, J. M. Molines, B. Barnier, and S. Bouillon (2009), Evaluation of high-resolution sea ice models on the basis of statistical and scaling properties of Arctic sea ice drift and deformation, J. Geophys. Res., 114, C08015, doi:10.1029/2008JC005182.

Haas, C., A. Pfaffling, S. Hendricks, L. Rabenstein, J.-L. Etienne, and I. Rigor (2008), Reduced ice thickness in Arctic transpolar drift favors rapid ice retreat, Geophys. Res. Lett., 35, L17501, doi:10.1029/2008GL034457.

Hall, R. T., and D. A. Rothrock (1981), Sea ice displacement from SEASAT synthetic aperture radar, J. Geophys. Res., 86(C11), 11,078-11,082.

Hopkins, M. A., and A. S. Thorndike (2006), Floe formation in Arctic sea ice, J. Geophys. Res., 111, C11S23, doi:10.1029/2005JC003352.

IPCC (2007), Climate Change 2007 - The Physical Science Basis, Contribution of Working Group I to the Fourth Assessment Report of the $I P C C$, Cambridge Univ. Press, Cambridge, U. K.

Korsnes, R., S. R. Souza, R. Donangelo, A. Hansen, M. Paczuski, and K. Sneppen (2004), Scaling in fracture and refreezing of sea ice, Physica A, $331(1-2), 291-296$.

Kwok, R. (1998), The RADARSAT Geophysical Processor System, in Analysis of SAR Data of the Polar Oceans: Recent Advances, edited by C. Tsatsoulis and R. Kwok, pp. 235-257, Springer-Verlag, New York.

Kwok, R. (2006), Contrasts in sea ice deformation and production in the Arctic seasonal and perennial ice zones, J. Geophys. Res., 111, C11S22, doi:10.1029/2005JC003246.

Kwok, R., and M. Coon (2006), Introduction to special section: Small-scale sea ice kinematics and dynamics, J. Geophys. Res., 111, C11S21, doi:10.1029/2006JC003877.

Kwok, R., E. Rignot, B. Holt, and R. Onstott (1992), Identification of sea ice types in spaceborne SAR data, J. Geophys. Res., 97(C2), 2391-2402

Kwok, R., D. A. Rothrock, H. L. Stern, and G. F. Cunningham (1995), Determination of the age distribution of sea ice from Lagrangian observations of ice motion, IEEE Trans. Geosci. Remote Sens., 33, 392-400.

Kwok, R., G. F. Cunningham, M. Wensnahan, I. Rigor, H. J. Zwally, and D. Yi (2009), Thinning and volume loss of the Arctic Ocean sea ice cover: 2003-2008, J. Geophys. Res., 114, C07005, doi:10.1029/2009JC005312.

Lindsay, R. W. (1998), Temporal variability of the energy balance of thick Arctic pack ice, J. Clim., 11, 313-333. 
Lindsay, R. W., and D. A. Rothrock (1995), Arctic sea ice leads from advanced very high resolution radiometer images, J. Geophys. Res., $100(\mathrm{C} 3), 4533-4544$.

Lindsay, R. W., and H. L. Stern (2003), The RADARSAT geophysical processor system: Quality of sea ice trajectory and deformation estimates, J. Atmos. Ocean. Technol., 20, 1333-1347.

Lindsay, R. W., J. Zhang, and D. A. Rothrock (2003), Sea-ice deformation rates from satellite measurements and in a model, Atmos. Ocean, 41(1), $35-47$.

Lindsay, R., J. Zhang, A. Schweiger, M. Steele, and H. Stern (2009), Arctic sea ice retreat in 2007 follows thinning trend, J. Clim., 22, doi:10.1175/ 2008JCLI2521.1.

Mandelbrot, B. B. (1982), The Fractal Geometry of Nature, W.H. Freeman, New York.

Marsan, D., H. Stern, R. Lindsay, and J. Weiss (2004), Scale dependence and localization of the deformation of Arctic sea ice, Phys. Rev. Lett., 93 , 17, doi:10.1103/PhysRevLett.93.178501.

Maslanik, J., C. Fowler, J. Stroeve, S. Drobot, J. Zwally, D. Yi, and W. Emery (2007), A younger, thinner Arctic ice cover: Increased potential for rapid, extensive sea-ice loss, Geophys. Res. Lett., 34, L24501, doi:10.1029/2007GL032043.

Meier, W. N., J. Stroeve, and F. Fetterer (2007), Whither Arctic sea ice? A clear signal of decline regionally, seasonally and extending beyond the satellite record, Ann. Glaciol., 46, 428-434, doi:10.3189/172756407782871170.

Nghiem, S. V., I. G. Rigor, D. K. Perovich, P. Clemente-Colón, J. W. Weatherly, and G. Neumann (2007), Rapid deduction of Arctic perennial sea ice, Geophys. Res. Lett., 34, L19504, doi:10.1029/2007GL031138.

Nye, J. F. (1973), Is there any physical basis for assuming linear viscous behavior for sea ice?, AIDJEX Bulletin 21, pp. 18-19. (Available at http://psc.apl.washington.edu/aidjex/)

Parkinson, C. L., and D. J. Cavalieri (2008), Arctic sea ice variability and trends, 1979-2006, J. Geophys. Res., 113, C07003, doi:10.1029/ 2007JC004558.

Rampal, P., J. Weiss, and D. Marsan (2007), Evidence for significant acceleration of Arctic sea ice drift over the last 25 years, Eos Trans. $A G U$, 88(52), Fall Meet Suppl., Abstract C11B-0438.
Rampal, P., J. Weiss, D. Marsan, R. Lindsay, and H. Stern (2008), Scaling properties of sea ice deformation from buoy dispersion analysis, J. Geophys. Res., 113, C03002, doi:10.1029/2007JC004143.

Rampal, P., J. Weiss, and D. Marsan (2009), Positive trend in the mean speed and deformation rate of Arctic sea ice, 1979-2007, J. Geophys. Res., 114, C05013, doi:10.1029/2008JC005066.

Rothrock, D. A., and A. S. Thorndike (1984), Measuring the sea ice floe size distribution, J. Geophys. Res., 89(C4), 6477-6486.

Stern, H. L., and R. E. Moritz (2002), Sea ice kinematics and surface properties from RADARSAT synthetic aperture radar during the SHEBA drift, J. Geophys. Res., 107(C10), 8028, doi:10.1029/2000JC000472.

Thorndike, A. S. (1986), Kinematics of Sea Ice, in The Geophysics of Sea Ice, NATO ASI Series, edited by N. Untersteiner, pp. 489-549, Plenum Press, New York.

Turcotte, D. L. (1992), Fractals and Chaos in Geology and Geophysics, Cambridge Univ. Press, Cambridge, U. K.

Weiss, J. (2001), Fracture and fragmentation of ice: A fractal analysis of scale invariance, Eng. Fract. Mech., 68, 1975-2012.

Weiss, J. (2003), Scaling of fracture and faulting of ice on Earth, Surv. Geophys., 24, 185-227.

Weiss, J., E. M. Schulson, and H. L. Stern (2007), Sea ice rheology from insitu, satellite and laboratory observations: Fracture and friction, Earth Planet. Sci. Lett., 255, 1-8.

Weiss, J., D. Marsan, and P. Rampal (2008), Space and time scaling laws induced by the multiscale fracturing of the Arctic sea ice cover, in Proceedings of the Symposium on Scaling in Solid Mechanics, International Union of Theoretical and Applied Mechanics, edited by B. Borodich, pp. 101-109, Springer, Cardiff.

Zhang, X., J. E. Walsh, J. Zhang, U. S. Bhatt, and M. Ikeda (2004), Climatology and interannual variability of arctic cyclone activity: 1948-2002, J. Clim., 17, 2300-2317.

R. W. Lindsay and H. L. Stern, Polar Science Center, Applied Physics Laboratory, University of Washington, 1013 NE 40th Street, Seattle, WA 98105, USA. (harry@apl.washington.edu) 